\title{
Discussion about differential equation of diffusion type in the Submarine Induced Polarization Electrical Prospecting
}

\author{
Wang Yuansheng ${ }^{1, a}$, Yan LinBo ${ }^{1, b}$, LU Guiying ${ }^{1,2, c}$
}

\author{
${ }^{1}$ School of Mechanics and Electrical Information, China University of Geo-Science, Wuhan Hubei, \\ 430074, \\ ${ }^{2}$ School of Sustainable Engineering and the Built Environment, Arizona State University, Tempe, AZ \\ 85287-0204, USA \\ a wangyshper@sohu.com, , ${ }^{\mathrm{b}} 913464653 @ q q . c o m,{ }^{\mathrm{C}} 75040781 @ q q . c o m$
}

Keywords: Maxwell equation; One-parameter transformation group; method of variable separation; Hermite equation;

Abstract: Hydrothermal sulfide ore in submarine mineral resources is a hot topic recent ocean exploration. From the fundamental equations of electromagnetic field, this paper first puts forward the model of $\nabla^{2} \hat{\mathrm{e}}-\mu \varepsilon \frac{\partial^{2} \hat{\mathrm{e}}}{\partial \mathrm{t}^{2}}-\mu\left(\sigma_{0}+\mathrm{Ae}^{-\mathrm{ac}}\right) \frac{\partial \hat{\mathrm{e}}}{\partial \mathrm{t}}=0$; Then, Analytical solution of the simplified nonlinear diffusion - heat conduction model $\mathrm{u}_{\mathrm{xx}}-\mathrm{pu}_{\mathrm{tt}}-(\mathrm{q}-\mathrm{wt}) \mathrm{u}_{\mathrm{t}}=0$ is given, which proves that there is no One-parameter transformation group makes the $\mathrm{p} \psi^{\prime \prime}+(\mathrm{q}-\mathrm{wt}) \psi^{\prime}+\psi(\mathrm{t})=0$ form remain the same. Finally, closed form analytical solution of the linear diffusion - heat conduction model $u_{x x}-\mathrm{pu}_{\mathrm{tt}}-\overline{\mathrm{q}} \mathrm{u}_{\mathrm{t}}=0$ is given, and the propagation rules of the electromagnetic field in the sea and the non ferromagnetic hydrothermal sulfide ore are simply explained. The theoretical study has important guiding significance to project implementation of frequency domain induced electric dipole drag system.

\section{Introduction}

Org Hydrothermal sulfide deposits in marine mineral resources are abundant, and the hydrothermal sulfide deposits can be developed in the background of the mid ocean ridge, the volcanic and the back arc basin. Research found that the seabed hydrothermal sulfide ore is mainly distributed in the low latitude Ocean near the ridge axial Valley and crater, where the depth general in hundreds of meters to 3700 meters, the temperature of about $20^{\circ} \mathrm{C}$ to $400^{\circ} \mathrm{C}$ and a pressure of about 20MPa-40MPa.In the ocean ridge setting, the depth of the hydrothermal activity are more concentrated in about 3000 meters; In the back arc basin tectonic environment, depth of the hydrothermal activity focus at 2800 meters; the depth of island arc volcanic tectonic environment of the hydrothermal activity are more concentrated in less than 2000 meters under the sea. Therefore, the 3000 meters in the ocean of poly metallic sulfide ore is a hot spot in the recent ocean exploration.

Nautilus Canada and Neptune Minerals and other companies as one of the pioneers of hydrothermal mineral exploration, mining is underway of seafloor hydrothermal sulfide deposit exploration, respectively in 2010 in the corresponding mining were test mining [1].

The transient electromagnetic method and induced polarization method are studied in the domestic. Transient electromagnetic method of theoretical research shows that drag and drop detection, the probe is not more than 50 meters from the height of the ore body [2]. Preliminary experimental results show that the induced polarization of metal sulfides can be effectively found by IP method. In order to reduce the supply current, the frequency domain induced polarization method is used to detect the $[3,4]$. Low frequency electromagnetic waves in seawater by diffusion heat conduction equation [5,6]. Seawater electromagnetic parameters including permeability $\mu$, conductivity $\sigma$, dielectric constant $\varepsilon$, they and seawater salinity, temperature, density. The sea water is a non ferromagnetic substance with a temperature of $17^{\circ} \mathrm{C}$ Seafloor hydrothermal sulfide Mineral 
type, metal content, temperature, and void density. In this paper, the influence of time varying conductivity on the marine electric field measurement was studied by using the single parameter transformation group (OPG) [7] method.

\section{Basic equation}

Induced polarization method is a method based on the difference of physical and electrochemical effects of rock and ore in the field of artificial electric field. The Maxwell equation of electromagnetic field in time domain is as follows[8,9]:

$$
\begin{gathered}
\nabla \times h=j+\frac{\partial d}{\partial t} \\
\nabla \times \hat{e}=-\frac{\partial b}{\partial t} \\
\nabla \bullet b=0
\end{gathered}
$$

$$
\nabla \bullet d=\rho
$$

The h,ê,b,d, j, $\rho$ in the formula are magnetic field strength, electric field strength, magnetic induction intensity, potential shift, current density, charge density and t is time coefficient.

The physical property equation is:

$$
\begin{gathered}
j=\sigma(t) \cdot \hat{e} \\
d=\varepsilon \cdot \hat{e} \\
\quad b=\mu \cdot h
\end{gathered}
$$

The $\sigma, \varepsilon, \mu$ in the form are conductivity, dielectric constant, permeability. Because the heat transfer problem has the form of the solution of $T=T_{0} \cdot e^{-a t}$, so set up:

$$
\sigma=A_{1} e^{-U_{1} /(K T)}+A_{2} e^{-U_{2} /(K T)}=\sigma_{0}+A e^{-a e^{t}}
$$

$A_{1}, A_{2}, U_{1}, U_{2}, \sigma_{0}, A, a$ are constants, and $\mathrm{K}$ is the Kelvin constant.

$$
\begin{gathered}
\nabla \times \nabla \times h-\nabla \times \frac{\partial d}{\partial t}=\nabla \times \nabla \times h-\nabla \times \frac{\partial(\varepsilon \hat{e})}{\partial t}=\nabla \times(\sigma \hat{e}) \\
\nabla \times \nabla \times \hat{e}+\mu \nabla \times \frac{\partial h}{\partial t}=0 \\
\nabla \times \nabla \times h-\varepsilon \nabla \times \frac{\partial \hat{e}}{\partial t}=\sigma \nabla \times \hat{e} \\
\nabla^{2} \hat{e}-\mu \varepsilon \frac{\partial^{2} \hat{e}}{\partial t^{2}}-\mu \sigma \frac{\partial \hat{e}}{\partial t}=0 \\
\nabla^{2} h-\mu \varepsilon \frac{\partial^{2} h}{\partial t^{2}}-\mu \sigma \frac{\partial h}{\partial t}=0
\end{gathered}
$$

Formula (12) (13) are diffusion heat conduction differential equation. When $\omega \leq 10^{5} \mathrm{~Hz}$ is $\mu \varepsilon \omega^{2}<<\mu \sigma \omega$, the conduction current plays an important role in $\nabla^{2} \hat{e}-\mu \sigma \frac{\partial \hat{e}}{\partial t}=0$, which is the diffusion equation. Steady state $\nabla^{2} \hat{e}=0$ this is the Poisson equation.

Since the $\sigma=\sigma_{0}+A e^{-a e^{t}}$ (A and a are constants) is a nonlinear time-varying factor, the differential equation of diffusion heat conduction can be studied by the method of Lie group [7]. 


\section{The OPG solutions of nonlinear equations}

That Equation (8) is substituted into equation (13) can be obtained:

$$
\nabla^{2} \hat{e}-\mu \varepsilon \frac{\partial^{2} \hat{e}}{\partial t^{2}}-\mu\left(\sigma_{0}+A e^{-a e^{t}}\right) \frac{\partial \hat{e}}{\partial t}=0
$$

Due to symmetry, the polarization field only Z component, application of $e^{t}=1+t+\sum_{n=2}^{\infty} \frac{t^{n}}{n !}$ series expression, neglecting the higher-order terms, there are:

$$
\hat{e}_{z z}-\mu \varepsilon \hat{e}_{t t}-\mu\left(\sigma_{0}+A-A a-A a t\right) \hat{e}_{t}=0
$$

By using the Semyon Quinn Love potential, the potential equation of the polarization field is rewritten as follows:

$$
u_{x x}-p u_{t t}-(q-w t) u_{t}=0
$$

The $u=u(x, t), \quad p=\mu \varepsilon, \quad q=\mu\left(\sigma_{0}+A-A a\right), \quad w=\mu A a$ are constants. using the method of separation of variables and set $u=u(x, t)=\phi(x) \psi(t)$, it can be concluded that:

$$
\begin{gathered}
\phi_{x x} \psi(t)-p \phi(x) \psi_{t t}-(q-w t) \phi(x) \psi_{t}=0 \\
\frac{\phi^{\prime \prime}}{\phi(x)}=\frac{p \psi^{\prime \prime}+(q-w t) \psi^{\prime}}{\psi(t)}=C
\end{gathered}
$$

$\mathrm{C}$ is a constant. $\frac{\phi^{\prime \prime}}{\phi(x)}=C$ can be obtained by $\phi^{\prime \prime}=C \phi(x)$, and then have :

$$
\phi(x)=g_{1} \cos (x)+g_{2} \sin (x), C=-1
$$

Those $g_{1}, g_{2}$ are constants. According to the formula $\frac{p \psi^{\prime \prime}+(q-w t) \psi^{\prime}}{\psi(t)}=-1$, it can get that :

$$
p \psi^{\prime \prime}+(q-w t) \psi^{\prime}+\psi(t)=0
$$

It is a nonlinear homogeneous equation, using the following OPG:

$$
\left\{\begin{array}{c}
t_{1}=t+\varepsilon \tau(t, \psi)+o\left(\varepsilon^{2}\right) \\
\psi_{1}=\psi+\varepsilon \eta(t, \psi)+o\left(\varepsilon^{2}\right)
\end{array}\right.
$$

It can the same form of : $p \psi_{1}^{\prime \prime}+\left(q-w t_{1}\right) \psi_{1}^{\prime}+\psi_{1}(t)=0$

$$
\begin{gathered}
\psi^{\prime \prime}=\left[-(q-w t) \psi^{\prime}-\psi(t)\right] / p=F\left(t, \psi, \psi^{\prime}\right) \quad \\
\left\{\eta_{t t}+\left(\eta_{\psi}-2 \tau_{t}\right) F-\tau \frac{\partial F}{\partial t}-\eta \frac{\partial F}{\partial \psi}-\eta_{t} \frac{\partial F}{\partial \psi^{\prime}}\right\}+\left\{2 \eta_{t \psi}-\tau_{t t}-3 \tau_{\psi} F-\left(\eta_{\psi}-\tau_{t}\right) \frac{\partial F}{\partial \psi^{\prime}}\right\} \psi^{\prime}+\left\{\eta_{\psi \psi}-2 \tau_{t \psi}+\tau_{\psi} \frac{\partial F}{\partial \psi^{\prime}}\right\}\left(\psi^{\prime}\right)^{2}+\tau_{\psi \psi}\left(\psi^{\prime}\right)^{3}=0
\end{gathered}
$$

Put the $\frac{\partial F}{\partial t}=\frac{w \psi^{\prime}}{p}, \frac{\partial F}{\partial \psi}=-\frac{1}{p}, \frac{\partial F}{\partial \psi^{\prime}}=\frac{w t-q}{p}$ into the formula, there is:

$\left\{\eta_{t t}+\left(\eta_{\psi}-2 \tau_{t}\right) \frac{\left[-(q-w t) \psi^{\prime}-\psi\right]}{p}-\frac{w \tau \psi^{\prime}}{p}+\frac{\eta}{p}-\frac{\eta_{t}(w t-q)}{p}\right\}+\left\{2 \eta_{t y}-\tau_{t t}-3 \tau_{\psi} \frac{\left[-(q-w t) \psi^{\prime}-\psi\right]}{p}-\frac{\left(\eta_{\psi}-\tau_{t}\right)(w t-q)}{p}\right\} \psi^{\prime}+\left\{\eta_{\psi y}-2 \tau_{t y}+\frac{\tau_{\psi}(w t-q)}{p}\right\}\left(\psi^{\prime}\right)^{2}+\tau_{\psi \psi}\left(\psi^{\prime}\right)^{\prime}=0$ 
In order to get the equation set:

$$
\begin{array}{ll}
\left(\psi^{\prime}\right)^{3}: & \tau_{\psi \psi}=0 \\
\psi \psi^{\prime}: & \frac{3 \tau_{\psi}}{p}=0 \Rightarrow \tau_{\psi}=0 \\
\left(\psi^{\prime}\right)^{2}: & \eta_{\psi \psi}-2 \tau_{t \psi}-2 \tau_{\psi}(w t-q) / p=0
\end{array}
$$

The formula (27') can be obtained:

$$
\eta_{\psi \psi}=0
$$

Then $\psi: \quad\left(\eta_{\psi}-2 \tau_{t}\right) / p=0 \quad$ can refer that:

$$
\begin{gathered}
\eta_{\psi}=2 \tau_{t} \\
\psi^{\prime}:-\left(\eta_{\psi}-2 \tau_{t}\right) \frac{(q-w t)}{p}-\frac{w \tau}{p}+2 \eta_{t \psi}-\tau_{t t}-\frac{\left(\eta_{\psi}-\tau_{t}\right)(w t-q)}{p}=0 \\
\psi^{0}: \quad \eta_{t t}-\frac{\eta_{t}(w t-q)}{p}+\frac{\eta}{p}=0
\end{gathered}
$$

Set $\tau=g_{3}+g_{4} t+g_{5} t^{2}+g_{6} t^{3}+\cdots$

$$
\begin{gathered}
3 \tau_{t t}-\tau_{t} \frac{(w t-q)}{p}-\frac{w \tau}{p}=0 \\
\eta_{t t}-\eta_{t} \frac{(w t-q)}{p}+\frac{\eta}{p}=0
\end{gathered}
$$

There is no OPG to make the equation $p \psi^{\prime \prime}+(q-w t) \psi^{\prime}+\psi(t)=0$ form unchanged. Similar to the $y^{\prime \prime}-2 x y^{\prime}+\mu y=0$ equation solution Hermite, to the equation $p \psi^{\prime \prime}+(q-w t) \psi^{\prime}+\psi(t)=0$, setting $g(t)=e^{\frac{q t-\frac{1}{2} w t^{2}}{P}} \psi^{\prime}$ it can refer that:

$$
\vartheta^{\prime}(t)=e^{\frac{q t-\frac{1}{2} w t^{2}}{p}} \psi^{\prime \prime}+\frac{q-w t}{p} \times e^{\frac{q t-\frac{1}{2} w t^{2}}{p}} \psi^{\prime}
$$

The equation $p \psi^{\prime \prime}+(q-w t) \psi^{\prime}+\psi(t)=0$ can be converted to:

$$
\frac{d}{d t}\left[e^{\frac{q t-\frac{1}{2} w t^{2}}{p}} \frac{d \psi}{d t}\right]+\frac{1}{p} e^{\frac{q t-\frac{1}{2} w t^{2}}{p}} \psi=0
$$

Therefore:

$$
\left\{\begin{array}{l}
\psi(t)=\sum_{n=0}^{\infty} l_{n} H_{n}(t) \\
l_{n}=\frac{1}{2^{n} \cdot n ! \sqrt{\pi}} \int_{-\infty}^{\infty} \psi(t) H_{n}(t) e^{\frac{q t-\frac{1}{2} w t^{2}}{p}} d t \\
H_{n}(t)=\frac{2^{n+1}}{\sqrt{2 \pi}} e^{t^{2}} \int_{0}^{\infty} \zeta^{n} e^{-\zeta^{2}} \cos \left(2 t \zeta-\frac{n \pi}{2}\right) d \zeta
\end{array}\right.
$$

$H_{n}(t)$ is the Hermite of the polynomial. Thus, the solution of the in situ potential equation (16) can be written as: 


$$
u=u(x, t)=\phi(x) \psi(t)=\left[g_{1} \cos (x)+g_{2} \sin (x)\right] \cdot\left[\sum_{n=0}^{\infty} l_{n} H_{n}(t)\right]
$$

\section{Analytical solution of linear diffusion type equation}

In the induced polarization method, the charge discharge frequency of the artificial electric field is low (e.g. $0.6 \mathrm{~Hz}, 9 \mathrm{~Hz}$ ), the discharge time is long enough and the full discharge, then the equation (14) can be simplified to the equation (37).

$$
\nabla^{2} \hat{e}-\mu \varepsilon \frac{\partial^{2} \hat{e}}{\partial t^{2}}-\mu \sigma_{0} \frac{\partial \hat{e}}{\partial t}=0
$$

In the same way, using the Semyon Quinn Love potential, the potential equation of the polarization field is as follows:

$$
\begin{gathered}
\hat{e}_{z z}-\mu \varepsilon \hat{e}_{t t}-\mu \sigma_{0} \hat{e}_{t}=0 \\
u_{x x}-p u_{t t}-\bar{q} u_{t}=0
\end{gathered}
$$

Among them $p=\mu \varepsilon, \bar{q}=\mu \sigma_{0}$.Using the method of separation of variables and setting $u=u(x, t)=\phi(x) \psi(t)$,it can get:

$$
\begin{gathered}
\phi_{x x} \psi(t)-p \phi(x) \psi_{t t}-\bar{q} \phi(x) \psi_{t}=0 \\
\frac{\phi^{\prime \prime}}{\phi(x)}=\frac{p \psi^{\prime \prime}+\bar{q} \psi^{\prime}}{\psi(t)}=C
\end{gathered}
$$

$\mathrm{C}$ is a constant. According to $\frac{\phi^{\prime \prime}}{\phi(x)}=C$ get $\phi^{\prime \prime}=C \phi(x)$, and there is:

$$
\phi(x)=g_{1} \cos (x)+g_{2} \sin (x), C=-1
$$

Those $g_{1}, g_{2}$ are Arbitrary constants. and According to $\frac{p \psi^{\prime \prime}+\bar{q} \psi^{\prime}}{\psi(t)}=-1$ get:

$$
p \psi^{\prime \prime}+\bar{q} \psi^{\prime}+\psi(t)=0
$$

This is a linear homogeneous differential equation with constant coefficients, which can be solved by Laplace transform.

$$
\begin{gathered}
p\left[s^{2} \Psi-s \psi(0)-\psi^{\prime}(0)\right]+\bar{q}[s \Psi-\psi(0)]+\Psi=0 \\
\Psi(s)=\frac{p\left[s \psi(0)+\psi^{\prime}(0)\right]+\bar{q} \psi(0)}{p s^{2}+\bar{q} s+1}
\end{gathered}
$$

$\psi(t)=L^{-1}\left\{\frac{p\left[s \psi(0)+\psi^{\prime}(0)\right]+\bar{q} \psi(0)}{p s^{2}+\bar{q} s+1}\right\}=\left\{\begin{array}{l}g_{3} e^{\lambda_{1} t}+g_{4} e^{\lambda_{2} t} \quad \lambda_{1} \neq \lambda_{2} \text { are real characteristic root } \\ \left(g_{5}+g_{6} t\right) e^{\lambda t} \quad \lambda \text { is multiple characteristic root } \\ e^{\operatorname{Re}\left(\lambda_{1}\right) t}\left(g_{7} \cos \left[\operatorname{Im}\left(\lambda_{1}\right) t\right]+g_{8} \sin \left[\operatorname{Im}\left(\lambda_{1}\right) t\right]\right) \lambda_{1,2} i s \text { conjugate complex roots }\end{array}\right.$

$\mathrm{g}_{3}, \mathrm{~g}_{4}, \mathrm{~g}_{5}, \mathrm{~g}_{6}, \mathrm{~g}_{7}, \mathrm{~g}_{8}$ are constants and $\lambda_{1,2}= \pm \sqrt{\overline{\mathrm{q}}^{2}-4 \mathrm{p}} \cdot \operatorname{Re}\left(\lambda_{1}\right), \operatorname{Im}\left(\lambda_{1}\right)$ are the real and imaginary parts of the complex. Thus, the solution of the in situ potential equation (39) can be written as:

$$
\begin{aligned}
& u=u(x, t)=\phi(x) \psi(t)=\left[g_{1} \cos (x)+g_{2} \sin (x)\right] \cdot L^{-1}\left\{\frac{p\left[s \psi(0)+\psi^{\prime}(0)\right]+\bar{q} \psi(0)}{p s^{2}+\bar{q} s+1}\right\} \\
& .=\left\{\begin{array}{l}
{\left[g_{1} \cos (x)+g_{2} \sin (x)\right] \cdot\left[g_{3} e^{\lambda_{1} t}+g_{4} e^{\lambda_{2} t}\right]} \\
{\left[g_{1} \cos (x)+g_{2} \sin (x)\right] \cdot\left(g_{5}+g_{6} t\right) e^{\lambda t}} \\
{\left[g_{1} \cos (x)+g_{2} \sin (x)\right] \cdot e^{\operatorname{Re}\left(\lambda_{1}\right) t}\left(g_{7} \cos \left[\operatorname{Im}\left(\lambda_{1}\right) t\right]+g_{8} \sin \left[\operatorname{Im}\left(\lambda_{1}\right) t\right]\right)}
\end{array}\right.
\end{aligned}
$$

It can get that: 


$$
\lambda_{p_{2}}= \pm \sqrt{\left(\mu \sigma_{0}\right)^{2}-4 \mu \varepsilon}= \pm \sqrt{\left(\mu \sigma_{0}\right)^{2}-4 \mu \varepsilon}
$$

Sea water as a non ferromagnetic material, when the temperature $17{ }^{\circ} \mathrm{C}$, Its conductivity is $_{\sigma=4.54-4.81(\Omega \cdot \mathrm{m})^{-1}}$, its permeability is $\mu=\mu_{0}=4 \pi \times 10^{-7}(\mathrm{H} / \mathrm{m})$, the sea water's dielectric constant $\varepsilon$ and salinity and temperature, by Debye expression, simplify take $\varepsilon=81 \varepsilon_{0}=81 \times 8.854 \times 10^{-12}(\mathrm{~F} / \mathrm{m})$, then $\lambda_{12} \approx \pm \sqrt{\left(\mu \sigma_{0}\right)^{2}}= \pm 6.3 \times 10^{-6}$. Therefore, in the non mining area, the potential variation of the induced electric field is:

$$
u=\left[g_{1} \cos (x)+g_{2} \sin (x)\right] \cdot\left\lfloor g_{3} e^{+6.3 \times 10^{-6} t}+g_{4} e^{-6.3 \times 10^{-6} t}\right\rfloor
$$

The variation of conductivity of seafloor hydrothermal sulfide ore is $\sigma=0.800-10.000(\Omega \cdot \mathrm{m})^{-1}$, The permeability of sulfide minerals containing $\mathrm{Cu}, \mathrm{Zn}$ and $\mathrm{Mn}$ is $\mu=\mu_{0}=4 \pi \times 10^{-7}(\mathrm{H} / \mathrm{m})$, and the dielectric constant of sulphide ore is $\varepsilon=(8-70) \varepsilon_{0}=(8-70) \times 8.854 \times 10^{-12}(\mathrm{~F} / \mathrm{m})$, so there is : $\lambda_{1{ }^{2}}= \pm \sqrt{\left(\mu \sigma_{0}\right)^{2}-4 \mu \mu} \approx \pm \sqrt{\left(\mu \sigma_{0}\right)^{2}}= \pm(1.0-12.6) \times 10^{-6}$ The potential variation of the induced electric field is in the non ferromagnetic one:

$$
u(x, t)=\left[g_{1} \cos (x)+g_{2} \sin (x)\right] \cdot\left[g_{3} e^{(1.0-12.6) \times 10^{-6} t}+g_{4} e^{-(1.0-12.6) \times 10^{-6} t}\right]
$$

\section{Conclusions}

In this paper, based on the basic equations of electromagnetic field, the electric field model $\nabla^{2} \hat{e}-\mu \varepsilon \frac{\partial^{2} \hat{e}}{\partial t^{2}}-\mu\left(\sigma_{0}+A e^{-a e^{t}}\right) \frac{\partial \hat{e}}{\partial t}=0$ is proposed. then an analytical solution is presented for the nonlinear diffusion heat conduction model $u_{x x}-p u_{t t}-(q-w t) u_{t}=0$.And it is proved that there is no OPG in the form of $p \psi^{\prime \prime}+(q-w t) \psi^{\prime}+\psi(t)=0$. Finally, the closed form analytical solution of the linear diffusion heat conduction model $u_{x x}-p u_{t t}-\bar{q} u_{t}=0$ is given. The transient propagation of the electric potential in the non ferromagnetic hydrothermal sulfide ore is briefly explained. On the magnetic field and magnetic potential model, It can also be used for similar processing. The research on the theory of the prospecting of the marine hydrothermal sulfide ore has important guiding significance for the engineering realization of the frequency domain induced dipole drag system. According to the actual situation, this paper simplifies the process, therefore, the above conclusions need to be verified by practice.

In addition, the analytical solution of $p \psi^{\prime \prime}+(q-w t) \psi^{\prime}+\psi(t)=0$ (equation (35) )must be deeply studied.

\section{References}

[1] Jing Chunlei. Regional geological background and ore controlling factors of poly metallic sulfide mineralization in the sea floor [D]. Qingdao: the first Institute of the State Oceanic Administration, 2012.6.

[2] Zhou Sheng, Deep drag type transient electromagnetic response rules [J]. Central South University Journal of (NATURAL SCIENCE EDITION):2012. Of 43 (2):1186-1189:605-610. .

[3] Xiao Hong Yang. He Jishan Frequency domain IP finite element numerical simulation [J]. Progress in Geophysics: 2008, 23 (4):605-610.

[4] Zhou Sheng, Deep drag type transient electromagnetic response rules [J]. Central South University Journal of (NATURAL SCIENCE EDITION):2012. Of 43 (2):136-140.

[5] Huang Junge, Ruan Bai Yao XJ.Underwater DC resistivity method simulation [J]. Geophysical exploration of computing technology, 2004, 26 (2) 
[6] Chen Yun, Wu Wu. Marine electromagnetism and its application [J]. Marine Science: 1992, 2:19-62.

[7] Pan Zuliang. Mathematical methods and applications of nonlinear problems [M]. Hangzhou: Zhejiang University press, 1998 44-80.

[8] Zhao Jingxiang, Wang Yanjun . Exploration geophysical electromagnetic method first volume [M]. Beijing: Geological Publishing House, 1992.120-154.

[9] Zhang Shengye, Pan Yuling. Application of the principle of Geophysics [M]. Wuhan: China University of Geosciences press, 2004 72-86. 